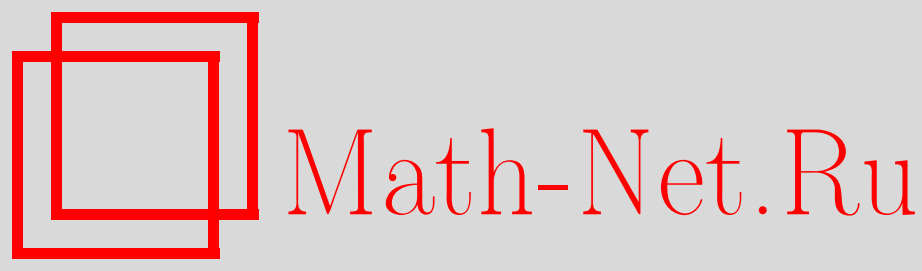

Б. Г. Конопельченко, Непрерывно-дискретные интегрируемые уравнения и преобразования Дарбу как деформации ассоциативных алгебр, ТMФ, 2009, том 159, номер 3, 502-514

DOI: https://doi.org/10.4213/tmf6368

Использование Общероссийского математического портала Math-Net.Ru подразумевает, что вы прочитали и согласны с пользовательским соглашением http://www . mathnet.ru/rus/agreement

Параметры загрузки:

IP : 54.209 .52 .79

26 апреля 2023 г., 08:29:49

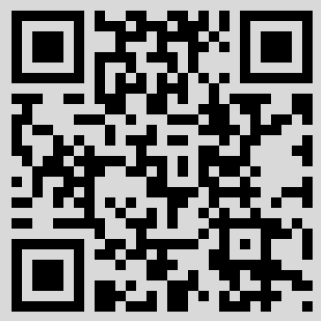




\title{
ФИЗИКА
}

Том 159, № 3

июнь, 2009

2009 г.

Б. Г. Конопельченко*

\section{НЕПРЕРЫВНО-ДИСКРЕТНЫЕ ИНТЕГРИРУЕМЫЕ УРАВНЕНИЯ И ПРЕОБРАЗОВАНИЯ ДАРБУ КАК ДЕФОРМАЦИИ АССОЦИАТИВНЫХ АЛГЕБР}

\begin{abstract}
Определяются и исследуются деформации структурных констант некоторого класса ассоциативных некоммутативных алгебр, порожденные алгебрами, вызывающими деформацию. Такие деформации управляются центральной системой. Подобная центральная система исследуется в случае, когда алгебра, вызывающая деформацию, представляет собой алгебру сдвигов. Представлены конкретные примеры деформаций трехмерных алгебр, управляемые дискретными и смешанными непрерывно-дискретными уравнением Буссинеска и уравнением ВДВВ. Показано, что теория преобразований Дарбу, по крайней мере для случая уравнения Буссинеска, полностью включается в предлагаемую схему деформаций.
\end{abstract}

Ключевые слова: ассоциативная алгебра, деформация, интегрируемое уравнение.

\section{1. ВВЕДЕНИЕ}

В настоящее время благодаря открытию уравнения ВДВВ [1], [2], его изящной формулировке, предложенной Дубровиным [3], [4] в рамках теории фробениусовых многообразий, и последующим обобщениям (см., например, работы [5], [6]) установлена глубокая связь между интегрируемыми системами и деформациями структурных констант ассоциативных алгебр.

Новый подход к теории деформаций структурных констант ассоциативных алгебр был недавно предложен в работах [7]-[11]. В отличие от теории фробениусовых многообразий и $F$-многообразий [3]-[6], где действие алгебры определяется на касательном расслоении, подход работ [7]-[11] формулируется в основном в терминах классической и квантовой механик и приводит к коизотропным и квантовым деформациям структурных констант. Одно из преимуществ данного подхода состоит в том, что он естественным образом допускает различные обобщения. Чтобы сформулировать их, кратко напомним некоторые факты о квантовых деформациях [10]. Подход состоит по существу из трех шагов:

${ }^{*}$ Dipartimento di Fisica, Universita del Salento and INFN, Sezione di Lecce, Lecce, Italy. E-mail: konopel@le.infn.it 
1) возьмем таблицу умножения ассоциативной алгебры в базисе $\mathbf{P}_{0}, \mathbf{P}_{1}, \ldots, \mathbf{P}_{N-1}$ со структурными константами $C_{j k}^{l}$, т.е.

$$
\mathbf{P}_{j} \mathbf{P}_{k}=C_{j k}^{l} \mathbf{P}_{l}
$$

и свяжем с ними набор операторов

$$
f_{j k}=-p_{j} p_{k}+C_{j k}^{l}\left(x^{0}, x^{1}, \ldots, x^{N-1}\right) p_{l}, \quad j, k=0,1, \ldots, N-1,
$$

где $x^{0}, x^{1}, \ldots, x^{N-1}$ - параметры деформации, $\mathbf{P}_{0}$ обозначает единичный элемент базиса и предполагается суммирование по повторяющимся индексам (от 0 до $N-1$ );

2 ) потребуем, чтобы операторы $p_{0}, p_{1}, \ldots, p_{N-1}$ и $x^{0}, x^{1}, \ldots, x^{N-1}$ были элементами алгебры Гейзенберга, т.е.

$$
\left[p_{j}, p_{k}\right]=0, \quad\left[x^{j}, x^{k}\right]=0, \quad\left[p_{j}, x^{k}\right]=\hbar \delta_{j}^{k}, \quad j, k=0,1, \ldots, N-1,
$$

где $\hbar$ - константа, а $\delta_{j}^{k}-$ символ Кронекера;

$3)$ наложим на функции $C_{j k}^{l}(x)$ следующее требование: система уравнений

$$
f_{j k}|\Psi\rangle=0, \quad j, k=0,1, \ldots, N-1,
$$

должна иметь нетривиальное общее решение (предписание Дирака), здесь $|\Psi\rangle$ принадлежит определенному линейному пространству.

Требование (4) приводит к системе уравнений

$$
\hbar \frac{\partial C_{j k}^{n}}{\partial x^{l}}-\hbar \frac{\partial C_{k l}^{n}}{\partial x^{j}}+C_{j k}^{m} C_{l m}^{n}-C_{k l}^{m} C_{j m}^{n}=0, \quad j, k, l, n=0,1, \ldots, N-1,
$$

назовем ее квантовой центральной системой (КЦС) [10]. Эта система управляет квантовыми деформациями структурных констант $C_{j k}^{l}\left(x^{0}, \ldots, x^{N-1}\right)$. В качестве частных случаев КЦС (5) содержит ориентированное уравнение ассоциативности, уравнение ВДВВ, уравнение Буссинеска и иерархии Гельфанда-Дикого и Кадомцева-Петвиашвили (КП) [10].

В настоящей работе мы дадим обобщение описанного выше подхода. Основная идея состоит в рассмотрении вместо алгебры Гейзенберга других алгебр, образованных базисными элементами $p_{0}, \ldots, p_{N-1}$ и параметрами деформации $x^{0}, \ldots, x^{N-1}$. Мы будем называть такие алгебры алгебрами, вызывающими дебормацию (АВД). Мы покажем, что при выборе АВД как алгебры разностей получается дискретная ЦС и далее дискретные интегрируемые уравнения и иерархии. АВД, которая содержит и подалгебру Гейзенберга, и подалгебру разностей, приводит к смешанным непрерывно-дискретным уравнениям и преобразованиям Дарбу для непрерывных систем.

В качестве иллюстрации общего подхода мы рассмотрим деформации трех- и четырехмерных ассоциативных алгебр. Соответствующая ЦС содержит, в частности, непрерывное уравнение Буссинеска, дискретное и смешанное непрерывно-дискретное уравнения Буссинеска и соответствующее преобразование Дарбу. Мы покажем, 
что преобразования Дарбу для уравнения Буссинеска полностью включаются в схему деформаций ассоциативных алгебр, представленную в настоящей работе. Еще одним показательным примером является уравнение ВДВВ в его дискретном и смешанном, т.е. непрерывно-дискретном, вариантах.

Работа построена следующим образом. В разделе 2 дается определение деформаций, порожденных АВД, и выводится общая ЦС. Непрерывные, дискретные и общие смешанные деформации Буссинеска рассматриваются в разделе 3. Раздел 4 посвящен исследованию уравнения ВДВВ, дискретному и смешанному уравнениям ВДВВ и их деформациям. Смешанные АВД и соответствующие преобразования Дарбу исследуются в разделе 5. Преобразования Дарбу для уравнения Буссинеска и деформации четырехмерной алгебры обсуждаются в разделе 6 .

\section{2. ДЕФОРМАЦИИ СТРУКТУРНЫХ КОНСТАНТ, ПОРОЖДЕННЫЕ АВД}

Рассмотрим конечномерную ассоциативную алгебру $A$ с единичным элементом $\mathbf{P}_{0}$ (или без него). Выберем базис $\mathbf{P}_{0}, \mathbf{P}_{1}, \ldots, \mathbf{P}_{N-1}$ и введем структурные константы согласно таблице умножения (1). Чтобы определить деформации $C_{j k}^{l}\left(x^{0}, x^{1}, \ldots, x^{N-1}\right)$ структурных констант, сначала отождествим элементы базиса $P_{j}$ и параметры деформации $x^{j}$ с элементами АВД. Далее введем операторы $f_{j k}$, заданные в $(2)$, и, наконец, потребуем, чтобы функции $C_{j k}^{l}(x)$ были таковы, чтобы эти операторы имели общее нетривиальное ядро или, эквивалентно, чтобы уравнения (4) имели нетривиальные общие решения (предписание Дирака). Если структурные константы $C_{j k}^{l}(x)$ таковы, что это требование выполнено, то будем говорить, что они определяют деформации, порожденные АВД. Требование (4) можно превратить в систему уравнений для $C_{j k}^{l}(x)$, которую мы будем называть ЦС для деформаций. Конкретный вид ЦС зависит от АВД. Например, для АВД Гейзенберга (3) ЦС есть КЦС (5).

В настоящей работе мы ограничимся ассоциативными алгебрами, которые имеют коммутативный базис, т.е. такими, в которых $C_{j k}^{l}(x)=C_{k j}^{l}(x)$. Для таких алгебр АВД должна иметь коммутативную подалгебру, порожденную элементами $p_{0}, \ldots, p_{N-1}$, т.е. $\left[p_{j}, p_{k}\right]=0$. Примерами алгебр, близких к АВД Гейзенберга (3), являются хорошо известные алгебра сдвигов

$$
\left[p_{j}, p_{k}\right]=0, \quad\left[x^{j}, x^{k}\right]=0, \quad\left[p_{j}, x^{k}\right]=\delta_{j}^{k} p_{j}, \quad j, k=0,1, \ldots, N-1,
$$

алгебра разностей

$$
\left[p_{j}, p_{k}\right]=0, \quad\left[x^{j}, x^{k}\right]=0, \quad\left[p_{j}, x^{k}\right]=\delta_{j}^{k}\left(\hat{I}+p_{j}\right), \quad j, k=0,1, \ldots, N-1,
$$

алгебра $q$-разностей

$$
\left[p_{j}, p_{k}\right]=0, \quad\left[x^{j}, x^{k}\right]=0, \quad\left[p_{j}, x^{k}\right]=\delta_{j}^{k}\left(\hat{I}+q x^{j} p_{j}\right), \quad j, k=0,1, \ldots, N-1
$$

и их смеси. Здесь $\hat{I}$ обозначает тождественный оператор.

Мы сконцентрируемся на алгебрах типов (3), (6), (7) и на АВД, содержащей их как подалгебры. Все эти алгебры можно представить единообразно как алгебры со 
следующими определяющими коммутационными соотношениями:

$$
\left[p_{j}, p_{k}\right]=0, \quad\left[x^{j}, x^{k}\right]=0, \quad\left[p_{j}, x^{k}\right]=\delta_{j}^{k}\left(\hat{I}+\varepsilon_{j} p_{j}\right), \quad j, k=0,1, \ldots, N-1,
$$

где $\varepsilon_{j}, j=0,1, \ldots, N-1$, произвольны. При $\varepsilon_{j} \rightarrow 0$ мы имеем подалгебру Гейзенберга, а при $\varepsilon_{j}=1$ - алгебру (7). Одна из реализаций такой АВД имеет вид алгебры разностей $p_{j}=\Delta_{j}$, где $\Delta_{j}=\left(T_{j}-1\right) / \varepsilon_{j}$ и $T_{j}\left(x^{k}\right)=x^{k}+\delta_{j}^{k} \varepsilon_{j}, j, k=0,1, \ldots, N-1$. При $\varepsilon_{j} \rightarrow 0$, очевидно, $\Delta_{j} \rightarrow \partial / \partial x^{j}$.

Для АВД (9) вывод ЦС основан на следующих тождествах. Первое имеет вид

$$
\left[p_{j}, \varphi(x)\right]=\Delta_{j} \varphi(x) \cdot\left(\hat{I}+\varepsilon_{j} p_{j}\right), \quad j=1,2, \ldots, N-1,
$$

где $\varphi(x)$ - произвольная функция,

$$
\begin{aligned}
\Delta_{j} \varphi\left(x^{0}, x^{1}, \ldots, x^{N-1}\right) & =\left(T_{j}-1\right) \varphi\left(x^{0}, x^{1}, \ldots, x^{N-1}\right), \\
T_{j} \varphi\left(x^{0}, \ldots, x^{j}, \ldots, x^{N-1}\right) & =\varphi\left(x^{0}, \ldots, x^{j}+\varepsilon_{j}, \ldots, x^{N-1}\right) .
\end{aligned}
$$

Второе тождество есть

$$
\begin{aligned}
\left(p_{j} p_{k}\right) p_{l}-p_{j}\left(p_{k} p_{l}\right)=- & p_{l} f_{j k}+p_{j} f_{k l}-T_{l} C_{j k}^{m} \cdot f_{l m}+T_{j} C_{k l}^{m} \cdot f_{j m}+ \\
+ & \left(\Delta_{l} C_{j k}^{n}-\Delta_{j} C_{k l}^{n}+T_{l} C_{j k}^{m} \cdot C_{l m}^{n}-T_{j} C_{k l}^{m} \cdot C_{j m}^{n}\right) p_{n} \\
& j, k, l=1,2, \ldots, N-1 .
\end{aligned}
$$

Из этого тождества следует, что

$$
\begin{aligned}
\left\{\left(p_{j} p_{k}\right) p_{l}-p_{j}\left(p_{k} p_{l}\right)\right\}|\Psi\rangle & =A_{k l j}^{n}(x) p_{n}|\Psi\rangle= \\
& =\left(\Delta_{l} C_{j k}^{n}-\Delta_{j} C_{k l}^{n}+T_{l} C_{j k}^{m} \cdot C_{l m}^{n}-T_{j} C_{k l}^{m} \cdot C_{j m}^{n}\right) p_{n}|\Psi\rangle
\end{aligned}
$$

где $|\Psi\rangle$ принадлежит линейному подпространству $H_{\Gamma}$, определяемому уравнениями (4), а $A_{k l j}^{n}$ - ассоциатор в алгебре $A$. Для ассоциативной алгебры левая часть уравнения (12) обращается в нуль; при условии, что подпространство $H_{\Gamma}$ не содержит элементов, линейных по $p_{j}|\Psi\rangle$, уравнение (12) выполнено, если и только если

$$
\Delta_{l} C_{j k}^{n}-\Delta_{j} C_{k l}^{n}+T_{l} C_{j k}^{m} \cdot C_{l m}^{n}-T_{j} C_{k l}^{m} \cdot C_{j m}^{n}=0, \quad j, k, l, n=0,1, \ldots, N-1 .
$$

Таким образом, имеем следующий факт.

ПредложЕниЕ. Структурные константы $C_{j k}^{l}(x)$ определяют деформации, порожденные АВД (9), если они подчиняются ЦС (13).

Подчеркнем, что ЦС (13) в действительности является следствием условий слабой ассоциативности

$$
\left\{\left(p_{j} p_{k}\right) p_{l}-p_{j}\left(p_{k} p_{l}\right)\right\}|\Psi\rangle=0 .
$$

В стандартных матричных обозначениях для матриц $C_{j}, A_{l j}$, задаваемых равенствами $\left(C_{j}\right)_{k}^{l}=C_{j k}^{l},\left(A_{l j}\right)_{k}^{n}=A_{k l j}^{n}$, ЦС (13) принимает вид

$$
A_{l j}^{d}:=\Delta_{l} C_{j}-\Delta_{j} C_{l}+C_{l} T_{l} C_{j}-C_{j} T_{j} C_{l}=0
$$


или

$$
A_{l j}^{d}:=\left(1+\varepsilon_{l} C_{l}\right) T_{l}\left(1+\varepsilon_{j} C_{j}\right)-\left(1+\varepsilon_{j} C_{j}\right) T_{j}\left(1+\varepsilon_{l} C_{l}\right)=0
$$

(в этих формулах нет суммирования по повторяющимся индексам).

В случае, когда все $\varepsilon_{j}=0$, имеем $T_{j}=1, \Delta_{j}=\partial / \partial x^{j}$, и ЦС (13) совпадает с КЦС (5). Если все $\varepsilon_{j}=1$, то получается чисто дискретная ЦС, рассмотренная в работе [11]. Здесь же мы имеем общую смешанную ЦС, которая будет весьма полезна при изучении соотношений между непрерывными и дискретными уравнениями, а также преобразований Дарбу. Заметим, что результаты работы [11], которые касаются соотношения между ассоциатором и дискретным тензором кривизны, верны и в общем случае.

\section{3. НЕПРЕРЫВНОЕ И ДИСКРЕТНОЕ УРАВНЕНИЯ БУССИНЕСКА}

Простой нетривиальный пример предложенной схемы отвечает трехмерной алгебре с единичным элементом и базисом $\mathbf{P}_{0}, \mathbf{P}_{1}, \mathbf{P}_{2}$. Таблица умножения содержит тривиальную часть $\mathbf{P}_{0} \mathbf{P}_{j}=\mathbf{P}_{j}, j=0,1,2$, а также соотношения

$$
\begin{aligned}
\mathbf{P}_{1}^{2} & =A \mathbf{P}_{0}+B \mathbf{P}_{1}+C \mathbf{P}_{2}, \\
\mathbf{P}_{1} \mathbf{P}_{2} & =D \mathbf{P}_{0}+E \mathbf{P}_{1}+G \mathbf{P}_{2}, \\
\mathbf{P}_{2}^{2} & =L \mathbf{P}_{0}+M \mathbf{P}_{1}+N \mathbf{P}_{2},
\end{aligned}
$$

где структурные константы $A, B, \ldots, N$ зависят только от параметров деформации $x^{1}, x^{2}$ вследствие цикличности переменной $x^{0}$, соответствующей единичному элементу $\mathbf{P}_{0}$. Из ЦС при $l=0$ легко получить, что $C_{j 0}^{l}=\delta_{j}^{l}$. В данном случае ЦС (13), порожденная АВД (9), имеет вид

$$
\begin{gathered}
\Delta_{1} D-\Delta_{2} A+A E_{1}+D G_{1}-D B_{2}-L C_{2}=0, \\
\Delta_{1} E-\Delta_{2} B+D_{1}+B E_{1}+E G_{1}-E B_{2}-M C_{2}=0, \\
\Delta_{1} G-\Delta_{2} C+C E_{1}+G G_{1}-A_{2}-G B_{2}-N C_{2}=0, \\
\Delta_{1} L-\Delta_{2} D+A M_{1}+D N_{1}-D E_{2}-L G_{2}=0, \\
\Delta_{1} M-\Delta_{2} E+L_{1}+B M_{1}+E N_{1}-E E_{2}-M G_{2}=0, \\
\Delta_{1} N-\Delta_{2} G+C M_{1}+G N_{1}-D_{2}-G E_{2}-N G_{2}=0,
\end{gathered}
$$

где $A_{j}:=T_{j} A, B_{j}:=T_{j} B$ и т.д. Данная ЦС, которая задает деформации структурных констант $A, B, \ldots, N$, является недоопределенной, а потому допускает калибровочный произвол.

Одна из калибровок, а именно $B=0, C=1, G=0$, обсуждалась в работе [10] в случае квантовых деформаций; было показано, что при этом КЦС редуцируется к уравнению Буссинеска. Рассмотрим здесь именно эту калибровку. Первые три уравнения в (17) дают

$$
L=\Delta_{1} D-\Delta_{2} A+A E_{1}, \quad M=\Delta_{1} E+D_{1}, \quad N=E_{1}-A_{2},
$$


а оставшаяся часть системы принимает вид

$$
\begin{gathered}
\left(\Delta_{1}^{2}-\Delta_{2}+E_{11}-E_{2}-A_{12}\right) E+2 \Delta_{1} D_{1}-\Delta_{2} A_{1}+A_{1} E_{11}=0, \\
\left(\Delta_{1}^{2}-\Delta_{2}+E_{11}-E_{2}-A_{12}\right) E+\left(-\Delta_{1} \Delta_{2}+\Delta_{1} E_{1}+D_{11}\right) A+\Delta_{1}\left(A E_{11}\right)=0, \\
\Delta_{1}\left(2 E_{1}-A_{2}\right)+D_{11}-D_{2}=0 .
\end{gathered}
$$

Эта ЦС определяет деформации Буссинеска структурных констант (16), порожденные АВД (9). Действительно, в случае $\varepsilon_{1}=\varepsilon_{2}=0$, т.е. для АВД Гейзенберга, ЦС (19) принимает вид $\left(A_{x_{j}}=\partial A / \partial x^{j}\right.$ и т.д.)

$$
\begin{gathered}
E_{x_{1} x_{1}}-E_{x_{2}}+2 D_{x_{1}}-A_{x_{2}}=0, \\
D_{x_{1} x_{1}}-D_{x_{2}}-A_{x_{1} x_{2}}+A E_{x_{1}}+(A E)_{x_{1}}=0, \quad 2 E-A=0,
\end{gathered}
$$

где все константы интегрирования выбраны равными нулю. Таким образом, имеем систему

$$
\frac{1}{2} A_{x_{1} x_{1}}-\frac{3}{2} A_{x_{2}}+2 D_{x_{1}}=0, \quad D_{x_{1} x_{1}}-D_{x_{2}}-A_{x_{1} x_{2}}+\frac{3}{4}\left(A^{2}\right)_{x_{1}}=0 .
$$

Исключая $D$, получаем уравнение Буссинеска

$$
A_{x_{2} x_{2}}+\frac{1}{3} A_{x_{1} x_{1} x_{1} x_{1}}-\left(A^{2}\right)_{x_{1} x_{1}}=0 .
$$

Это уравнение определяет квантовые деформации структурных констант (16) при

$$
\begin{gathered}
B=0, \quad C=1, \quad G=0, \\
L=D_{x_{1}}-A_{x_{2}}+\frac{A^{2}}{2}, \quad M=\frac{1}{2} A_{x_{1}}+D, \quad N=-\frac{1}{2} A,
\end{gathered}
$$

где $A$ и $D$ находятся из (21). В чисто дискретном случае $\varepsilon_{1}=\varepsilon_{2}=1$ ЦС (19) представляет собой дискретный вариант системы Буссинеска (21). Она определяет дискретные деформации тех же структурных констант.

Имеются также смешанные случаи. Первый из них задается соотношениями $\varepsilon_{1}=0, \varepsilon_{2}=1\left(T_{1}=1, \Delta_{1}=\partial / \partial x^{1}, \Delta_{2}=T_{2}-1\right)$. ЦС (19) имеет вид

$$
\begin{gathered}
E_{x_{1} x_{1}}+2 D_{x_{1}}-(1+E) \Delta_{2}(E+A)=0 \\
D_{x_{1} x_{1}}-D_{x_{2}}+A E_{x_{1}}+(A E)_{x_{1}}-\Delta_{2} A_{x_{1}}-D \Delta_{2}(E+A)=0 \\
\left(2 E-A_{2}\right)_{x_{1}}-\Delta_{2} D_{2}=0 .
\end{gathered}
$$

Второй случай соответствует $\varepsilon_{1}=1, \varepsilon_{2}=0\left(\Delta_{1}=T_{1}-1, T_{2}=1, \Delta_{2}=\partial / \partial x^{2}\right)$, а ЦС принимает вид

$$
\begin{gathered}
\Delta_{1}^{2} E+\left(E_{11}-E-A_{1}\right) E+A_{1} E_{11}+2 \Delta_{1} D_{1}-\left(E+A_{1}\right)_{x_{2}}=0 \\
\Delta_{1}^{2} D+\left(E_{11}-E-A_{1}\right) D+A \Delta_{1} E_{1}+\Delta_{1}\left(A E_{1}\right)+D_{11} A-\Delta_{1} A_{x_{2}}-D_{x_{2}}=0 \\
\Delta_{1}\left(2 E_{1}-A\right)+D_{11}-D=0 .
\end{gathered}
$$

Уравнения (4) дают следующие линейные задачи для ЦС (19):

$$
\left(\Delta_{2}-\Delta_{1}^{2}+A\right)|\Psi\rangle=0, \quad\left(\Delta_{1} \Delta_{2}-E \Delta_{1}-D\right)|\Psi\rangle=0
$$


или, эквивалентно,

$$
\left(\Delta_{2}-\Delta_{1}^{2}+A\right)|\Psi\rangle=0, \quad\left(\Delta_{1}^{3}-(E+A) \Delta_{1}-\left(D+\Delta_{1} A\right)\right)|\Psi\rangle=0 .
$$

Можно проверить, что уравнение $f_{22}|\Psi\rangle=0$ является следствием уравнений (25).

В квантовом случае $\left(\Delta_{j}=\partial / \partial x^{j}\right)$ система (26) является хорошо известной линейной системой для непрерывного уравнения Буссинеска [12]. Заметим, что в этом случае третье уравнение $f_{22}|\Psi\rangle=0$, т.е. $\left(\Delta_{2}^{2}-L-D \Delta_{1}+A / 2\right)|\Psi\rangle=0$, является следствием двух уравнений (26).

В чисто дискретном случае уравнения (26) представляют собой линейные задачи для дискретного уравнения Буссинеска. Первый смешанный случай, рассмотренный выше, можно трактовать как уравнение Буссинеска с дискретным временем. Второй случай соответствует непрерывному изоспектральному потоку для разностной задачи третьего порядка, т.е. некоторому виду разностного уравнения Буссинеска с непрерывным временем.

\section{4. УРАВНЕНИЕ ВДВВ, ДИСКРЕТНОЕ И НЕПРЕРЫВНО-ДИСКРЕТНОЕ УРАВНЕНИЯ ВДВВ}

Еще одна интересная редукция ЦС (19) соответствует связям $C=1, G=0$, $N=0$. В этом случае ЦС есть

$$
\begin{gathered}
\Delta_{1} D-\Delta_{2} A+A E_{1}-D B_{2}-L=0, \\
\Delta_{1} E-\Delta_{2} B+D_{1}+B E_{1}+E G_{1}-E B_{2}-M=0, \\
\Delta_{1} L-\Delta_{2} D+A M_{1}-D E_{2}=0, \\
\Delta_{1} M-\Delta_{2} E+L_{1}+B M_{1}-E E_{2}=0, \\
E_{1}-A_{2}=0, \quad M_{1}-D_{2}=0 .
\end{gathered}
$$

Из третьего и шестого уравнений этой системы следует существование таких функций $U$ и $V$, что

$$
A=U_{1}, \quad E=U_{2}, \quad D=V_{1}, \quad M=V_{2} .
$$

Подставляя выражения для $L$ и $M$ из первых двух уравнений системы (27), т.е.

$$
\begin{aligned}
L & =\Delta_{1} V_{1}-\Delta_{2} U_{1}+U_{1} U_{12}-V_{1} B_{2}, \\
M & =\Delta_{1} U_{2}-\Delta_{2} B+V_{11}+B U_{12}-U_{2} B_{2},
\end{aligned}
$$

в оставшиеся уравнения системы, получаем следующие три уравнения:

$$
\begin{gathered}
\Delta_{1} U_{1}-\Delta_{2} B+V_{11}+B U_{12}-U_{2} B_{2}-V_{2}=0 \\
\left(\Delta_{1}^{2}-\Delta_{2}\right) V_{1}-\Delta_{1} \Delta_{2} U_{1}+\Delta_{1}\left(U_{1} U_{12}\right)-\Delta_{1}\left(V_{1} B_{2}\right)-V_{1} U_{22}+U_{1} V_{12}=0 \\
\Delta_{1}\left(V_{2}+V_{11}\right)-\Delta_{2}\left(U_{2}+U_{11}\right)+U_{11} U_{12}-U_{2} U_{22}-V_{11} B_{12}+B V_{12}=0 .
\end{gathered}
$$

Решение этой системы вместе с формулами (28) определяет деформации структурных констант $A, B, \ldots$, порожденные АВД (9). 
В чисто непрерывном случае $\varepsilon_{1}=\varepsilon_{2}=0$ система (29) принимает вид

$$
\begin{gathered}
U_{x_{1}}-B_{x_{2}}=0, \quad V_{x_{1}}-U_{x_{2}}=0, \\
\left(V_{x_{1}}+U_{x_{2}}+U^{2}-V B\right)_{x_{1}}-V_{x_{2}}=0 .
\end{gathered}
$$

Из этой системы, выражающей три закона сохранения, вытекает существование такой функции $F$, что

$$
U=F_{x_{1} x_{1} x_{2}}, \quad V=F_{x_{1} x_{2} x_{2}}, \quad B=F_{x_{1} x_{1} x_{1}} .
$$

В терминах $F$ система (30) принимает вид

$$
F_{x_{2} x_{2} x_{2}}-\left(F_{x_{1} x_{1} x_{2}}\right)^{2}+F_{x_{1} x_{1} x_{1}} F_{x_{1} x_{2} x_{2}}=0 .
$$

Это знаменитое уравнение ВДВВ [1], [2]. Хорошо известно, что оно описывает деформации трехмерной алгебры (16) при редукции $C=1, G=0, N=0$ [3]-[10]. Таким образом, система (29) представляет собой обобщение уравнения ВДВВ на случай деформаций той же алгебры, порожденных АВД (9).

В чисто дискретном случае $\varepsilon_{1}=\varepsilon_{2}=1$ система (29) дает чисто дискретный вариант уравнения ВДВВ. В первом смешанном случае $\varepsilon_{1}=0, \varepsilon_{2}=1$ имеем систему

$$
\begin{gathered}
U_{x_{1}}-\Delta_{2}(B+V)-U_{2} \Delta_{2} B=0, \\
\left(V_{x_{1}}+U U_{2}-V B_{2}\right)_{x_{1}}-\Delta_{2}\left(V+U_{x_{1}}\right)-V U_{22}+U V_{2}=0, \\
\left(V+V_{2}\right)_{x_{1}}-\Delta_{2}\left(U+U_{2}+U U_{2}\right)-V B_{2}+B V_{2}=0,
\end{gathered}
$$

а в случае $\varepsilon_{1}=1, \varepsilon_{2}=0$ получаем

$$
\begin{gathered}
\Delta_{1} U_{1}+B \Delta_{1} U+V_{11}-V-B_{x_{2}}=0 \\
\Delta_{1}\left(\Delta_{1} V_{1}-U_{1 x_{2}}+U_{1}^{2}-V_{1} B\right)-V_{1} U+U_{1} V_{1}-V_{1 x_{2}}=0, \\
\Delta_{1}\left(V+V_{11}\right)+U_{11}^{2}-U^{2}-V_{11} B_{1}+B V_{1}-\left(U+U_{11}\right)_{x_{2}}=0 .
\end{gathered}
$$

Уравнения $f_{j k}|\Psi\rangle=0$ для системы (29) в координатном представлении $p_{j}=\Delta_{j}$ имеют вид

$$
\begin{gathered}
\left(\Delta_{2}-\Delta_{1}^{2}+B \Delta_{1}+A\right)|\Psi\rangle=0, \\
\left(\Delta_{1} \Delta_{2}-E \Delta_{1}-D\right)|\Psi\rangle=0, \\
\left(\Delta_{2}^{2}-M \Delta_{1}-L\right)|\Psi\rangle=0 .
\end{gathered}
$$

Эта система в свою очередь эквивалентна следующей:

$$
\begin{gathered}
\left(\Delta_{1}^{3}-B \Delta_{1}^{2}-\left(\Delta_{1} B+A_{1}+E\right) \Delta_{1}-\left(\Delta_{1} A+D\right)\right)|\Psi\rangle=0, \\
\left(\Delta_{2}-\Delta_{1}^{2}+B \Delta_{1}+A\right)|\Psi\rangle=0, \quad\left(\Delta_{2}^{2}-M \Delta_{1}-L\right)|\Psi\rangle=0 .
\end{gathered}
$$

Условия совместности для приведенных выше линейных задач эквивалентны “дискретизованным" системам уравнений ВДВВ (27) или (29). В частности, в чисто непрерывном случае задачи (35) в координатном представлении они имеют хорошо известный вид [3], [4]

$$
\begin{aligned}
& \Psi_{x_{1} x_{1}}=F_{x_{1} x_{1} x_{2}} \Psi+F_{x_{1} x_{1} x_{1}} \Psi_{x_{1}}+\Psi_{x_{2}}, \\
& \Psi_{x_{1} x_{2}}=F_{x_{1} x_{2} x_{2}} \Psi+F_{x_{1} x_{1} x_{2}} \Psi_{x_{1}}, \\
& \Psi_{x_{2} x_{2}}=F_{x_{2} x_{2} x_{2}} \Psi+F_{x_{1} x_{2} x_{2}} \Psi_{x_{1}} .
\end{aligned}
$$


Первое из этих уравнений есть редукция линейной задачи

$$
\Psi_{x_{2}}=\Psi_{x_{1} x_{1}}+V \Psi_{x_{1}}+U \Psi
$$

связанной с обобщенной (КП-мКП)-иерархией. Таким образом, уравнение ВДВВ является очень специальной редукцией этой иерархии, стационарной по отношению к временам $x_{3}, x_{4}$ (по поводу похожих результатов см. работу [13]).

\section{5. СМЕШАНННЫЕ АВД И ПРЕОБРАЗОВАНИЯ ДАРБУ}

При $N \geqslant 4$ ЦС (13) (или (15)), порожденная АВД (9), содержит намного больше смешанных случаев, находящихся между случаями чисто непрерывных и чисто дискретных уравнений. Ситуация с $N-1$ непрерывной и одной дискретной переменными, т.е. случай $\varepsilon_{0}=\varepsilon_{1}=\cdots=\varepsilon_{N-2}=0$ и $\varepsilon_{N-1}=1$, представляет особый интерес. Коммутационные соотношения (9), определяющие АВД данного типа, можно представить как

$$
\begin{gathered}
{\left[p_{j}, p_{k}\right]=0, \quad\left[x^{j}, x^{k}\right]=0, \quad\left[p_{j}, x^{k}\right]=\hbar \delta_{j}^{k}} \\
{\left[p_{j}, T\right]=0, \quad\left[x^{j}, n\right]=0, \quad\left[p_{j}, n\right]=0, \quad[T, n]=T, \quad j, k=0,1, \ldots, N-2,}
\end{gathered}
$$

где $T=p_{N-1}+1$, а $n$ - дискретная переменная, связанная с $T$. Таблица умножения в общем случае имеет вид

$$
\begin{array}{rlr}
\mathbf{P}_{j} \mathbf{P}_{k} & =\sum_{l=0}^{N-2} C_{j k}^{l} \mathbf{P}_{l}+C_{j k}^{N-1} T, & j, k=0,1, \ldots, N-2, \\
\mathbf{T P}_{k} & =\sum_{l=0}^{N-2} C_{N-1, k}^{l} \mathbf{P}_{l}+C_{N-1, k}^{N-1} T, & k=0,1, \ldots, N-2, \\
\mathbf{T}^{2} & =\sum_{l=0}^{N-2} C_{N-1, N-1}^{l} \mathbf{P}_{l}+C_{N-1, N-1}^{N-1} T . &
\end{array}
$$

Замена базиса $p_{N-1} \rightarrow T$ в этой таблице выполнена для удобства. Если имеют место связи $C_{j k}^{N-1}=0, j, k=0,1, \ldots, N-2$, то такая алгебра имеет специальный смысл: она содержит подалгебру, линейно порожденную элементами $p_{0}, p_{1}, \ldots, p_{N-2}$. Что касается ЦС, то можно показать, что она разделяется на две части

$$
\frac{\partial C_{j}}{\partial x^{k}}-\frac{\partial C_{k}}{\partial x^{j}}+\left[C_{k}, C_{j}\right]=0, \quad j, k=0,1, \ldots, N-2,
$$

и

$$
\frac{\partial C_{N-1}}{\partial x^{l}}+C_{l} C_{N-1}-C_{N-1} T C_{l}=0, \quad l=0,1, \ldots, N-2,
$$

где $T C_{l}\left(x^{1}, x^{2}, \ldots, x^{N-2}, n\right)=C_{l}\left(x^{1}, x^{2}, \ldots, x^{N-2}, n+1\right)$. Отметим отличие от ЦС (13), возникающее в силу замены $p_{N-1} \rightarrow T$. Условия совместности для уравнений (43) выполнены в силу того, что матрица $T C_{j}$, как и $C_{j}$, подчиняется уравнениям $(42)$ для всех $j=0,1, \ldots, N-2$. 
Несложно показать, что система (42) содержит замкнутую подсистему для первых главных $(N-2) \times(N-2)$-миноров $\widetilde{C}_{j}$ матрицы $C_{j}\left(\right.$ т.е. $\left(\widetilde{C}_{j}\right)_{k}^{l}=C_{j k}^{l}, l, k=$ $0,1, \ldots, N-2)$ :

$$
\frac{\partial \widetilde{C}_{j}}{\partial x^{k}}-\frac{\partial \widetilde{C}_{k}}{\partial x^{j}}+\left[\widetilde{C}_{k}, \widetilde{C}_{j}\right]=0, \quad j, k=0,1, \ldots, N-2
$$

Эта подсистема определяет интегрируемую систему дифференциальных уравнений, которая совпадает с КЦС (5) при $j, k, l, n=0,1, \ldots, N-2$ и при независимых переменных $x^{1}, x^{2}, \ldots, x^{N-2}$. Остальная часть системы (42) и уравнений (43) определяют преобразование $\widetilde{C}_{l} \rightarrow T \widetilde{C}_{l}$, которое превращает решения системы (14) в решения этой же системы. Таким образом, уравнения (42), (43) определяют преобразование Дарбу для непрерывной системы (44).

Для иллюстрации этой общей схемы рассмотрим простейший случай $N=4$, т.е. четырехмерную ассоциативную алгебру с коммутативным базисом $\mathbf{P}_{0}, \mathbf{P}_{1}, \mathbf{P}_{2}, \mathbf{T}$. Таблица умножения имеет вид (мы приводим нетривиальную ее часть)

$$
\begin{aligned}
\mathbf{P}_{1}^{2} & =A \mathbf{P}_{0}+B \mathbf{P}_{1}+C \mathbf{P}_{2}, \\
\mathbf{P}_{1} \mathbf{P}_{2} & =D \mathbf{P}_{0}+E \mathbf{P}_{1}+G \mathbf{P}_{2}, \\
\mathbf{P}_{2}^{2} & =L \mathbf{P}_{0}+M \mathbf{P}_{1}+N \mathbf{P}_{2}, \\
\mathbf{P}_{1} \mathbf{T} & =a_{1} \mathbf{P}_{0}+a_{2} \mathbf{P}_{1}+a_{3} \mathbf{P}_{2}+a_{4} \mathbf{T}, \\
\mathbf{P}_{2} \mathbf{T} & =b_{1} \mathbf{P}_{0}+b_{2} \mathbf{P}_{1}+b_{3} \mathbf{P}_{2}+b_{4} \mathbf{T}, \\
\mathbf{T}^{2} & =c_{1} \mathbf{P}_{0}+c_{2} \mathbf{P}_{1}+c_{3} \mathbf{P}_{2}+c_{4} \mathbf{T} .
\end{aligned}
$$

Матрица $C_{0}=1$, а матрицы $C_{1}, C_{2}, C_{3}$ таковы:

$$
C_{1}=\left(\begin{array}{cccc}
0 & A & D & a_{1} \\
1 & B & E & a_{2} \\
0 & C & G & a_{3} \\
0 & 0 & 0 & a_{4}
\end{array}\right), \quad C_{2}=\left(\begin{array}{cccc}
0 & D & L & b_{1} \\
0 & E & M & b_{2} \\
1 & G & N & b_{3} \\
0 & 0 & 0 & b_{4}
\end{array}\right), \quad C_{3}=\left(\begin{array}{cccc}
0 & a_{1} & b_{1} & c_{1} \\
0 & a_{2} & b_{2} & c_{2} \\
0 & a_{3} & b_{3} & c_{3} \\
1 & a_{4} & b_{4} & c_{4}
\end{array}\right)
$$

Уравнение (44) при $j=1, k=2$ эквивалентно чисто непрерывной системе (17). Оставшаяся часть уравнений (42) принимает вид

$$
\begin{gathered}
a_{1 x_{2}}-b_{1 x_{1}}+D a_{2}+L a_{3}-A b_{2}-D b_{3}+b_{1} a_{4}-a_{1} b_{4}=0, \\
a_{2 x_{2}}-b_{2 x_{1}}+E a_{2}+M a_{3}-B b_{2}-E b_{3}-b_{1}+b_{2} a_{4}-a_{2} b_{4}=0, \\
a_{3 x_{2}}-b_{3 x_{1}}+G a_{2}+N a_{3}-C b_{2}-G b_{3}+a_{1}+b_{3} a_{4}-a_{3} b_{4}=0, \\
a_{4 x_{2}}-b_{4 x_{1}}=0 .
\end{gathered}
$$

Матричные уравнения (43) эквивалентны 24 скалярным дифференциальным уравнениям. Анализ этих уравнений и уравнений (46) позволяет заключить, что не все они независимы. Далее, из этого следует, что все эти уравнения в принципе позволяют восстановить $a_{j}, b_{j}, c_{j}, j=1,2,3,4$, и в конце концов $T A, T B$ и т.д. в терминах $A, B$ и т.д. 


\section{6. ПРЕОБРАЗОВАНИЕ ДАРБУ ДЛЯ УРАВНЕНИЯ БУССИНЕСКА КАК ДИСКРЕТНАЯ ДЕФОРМАЦИЯ}

Приведем решение выписанных выше уравнений в случае редукции Буссинеска $B=0, C=1, G=0$. В этом случае $L=D_{x_{1}}-A_{x_{2}}+A^{2} / 2, M=A_{x_{1}} / 2+D$, $N=-A / 2$ (см. раздел 3 ). Начнем с двух уравнений из системы (43), которые в калибровке Буссинеска имеют вид

$$
a_{4 x_{1}}+a_{4}^{2}-b_{4}-T A=0, \quad b_{4 x_{1}}+a_{4} b_{4}-\frac{1}{2} a_{4} T A-T D=0 .
$$

Из последнего уравнения в (46) следует, что существует такая функция $\varphi$, что

$$
a_{4}=(\ln \varphi)_{x_{1}}, \quad b_{4}=(\ln \varphi)_{x_{2}} .
$$

Подставляя эти выражения в (47), получаем уравнения

$$
\varphi_{x_{2}}=\varphi_{x_{1} x_{1}}-T A \varphi, \quad \varphi_{x_{1} x_{2}}=\frac{1}{2} T A \varphi_{x_{1}}+T D \varphi,
$$

которые являются просто линейными задачами (26) для непрерывного уравнения Буссинеска относительно сдвинутых решений $T A$ и $T D$ (это весьма полезно в вычислениях). Чтобы упростить их, рассмотрим специальный случай $a_{3}=1, c_{3}=1$. Весьма долгий анализ, который мы опускаем, показывает, что при таком выборе ЦС (46) имеет решение

$$
\begin{gathered}
a_{1}=A-(\ln \widetilde{\Psi})_{x_{1} x_{1}}+(\ln \widetilde{\Psi})_{x_{1}}(\ln \varphi)_{x_{1}}, \quad a_{2}=-(\ln (\widetilde{\Psi} \varphi))_{x_{1}}, \\
a_{3}=1, \quad a_{4}=(\ln \varphi)_{x_{1}}, \\
b_{1}=D-(\ln \widetilde{\Psi})_{x_{1} x_{2}}+(\ln \widetilde{\Psi})_{x_{1}}(\ln \varphi)_{x_{2}}, \quad b_{2}=\frac{1}{2} A-(\ln \varphi)_{x_{2}}, \\
b_{3}=-(\ln \widetilde{\Psi})_{x_{1}}, \quad b_{4}=(\ln \varphi)_{x_{2}}, \\
c_{1}=A-(\ln \widetilde{\Psi})_{x_{1} x_{1}}-(\ln \widetilde{\Psi})_{x_{1}}(\ln \varphi)_{x_{1}}+(\ln \widetilde{\Psi})_{x_{1}} \frac{T \varphi}{\varphi}, \\
c_{2}=-(\ln (\widetilde{\Psi} \varphi))_{x_{1}}-\frac{T \varphi}{\varphi}, \quad c_{3}=1, \quad c_{4}=\frac{T \varphi}{\varphi},
\end{gathered}
$$

причем

$$
T A=A-2(\ln \widetilde{\Psi})_{x_{1} x_{1}}, \quad T D=D+\frac{1}{2}(\ln \widetilde{\Psi})_{x_{1} x_{1} x_{1}}-\frac{3}{2}(\ln \widetilde{\Psi})_{x_{1} x_{2}},
$$

где $\widetilde{\Psi}$ - решение линейных задач для уравнения Буссинеска

$$
\Psi_{x_{2}}=\Psi_{x_{1} x_{1}}-A \Psi, \quad \Psi_{x_{1} x_{2}}=\frac{1}{2} A \Psi_{x_{1}}+D \Psi,
$$

а $\varphi$ - решение уравнений (49).

Формулы (51) представляют собой хорошо известное преобразование Дарбу для системы уравнений Буссинеска (22) (см., например, [14]). Напомним, что в терминах $\tau$-функции, определяемой формулами

$$
A=-2(\ln \tau)_{x_{1} x_{1}}, \quad D=-\frac{3}{2}(\ln \tau)_{x_{1} x_{2}}+\frac{1}{2}(\ln \tau)_{x_{1} x_{1} x_{1}},
$$

преобразование Дарбу выглядит очень просто: $T \tau=\widetilde{\Psi} \tau$. 
Итак, мы построили деформации структурных констант из таблицы умножения (45), параметризованные двумя непрерывными переменными $x^{1}, x^{2}$ и одной дискретной переменной $n$. Они управляются системой уравнений Буссинеска (22), формулами (18), (50) и преобразованиями (51). С точки зрения интегрируемых систем соответствующая ЦС представляет собой композицию уравнения Буссинеска и его преобразования Дарбу.

Эти деформации имеют одно важное алгебраическое свойство. Используя формулы (50), можно показать, что линейные задачи $f_{j k}|\Psi\rangle=0$ эквивалентны следующим уравнениям:

$$
\left(p_{2}-p_{1}^{2}+A p_{0}\right)|\Psi\rangle=0, \quad\left(p_{1} p_{2}-\frac{1}{2} A p_{1}-D p_{0}\right)|\Psi\rangle=0
$$

и

$$
\begin{aligned}
\left(p_{1}-a_{4} p_{0}\right)\left(T-p_{1}+(\ln \widetilde{\Psi})_{x_{1}} p_{0}\right)|\Psi\rangle & =0, \\
\left(p_{2}-b_{4} p_{0}\right)\left(T-p_{1}+(\ln \widetilde{\Psi})_{x_{1}} p_{0}\right)|\Psi\rangle & =0, \\
\left(T-c_{4} p_{0}\right)\left(T-p_{1}+(\ln \widetilde{\Psi})_{x_{1}} p_{0}\right)|\Psi\rangle & =0 .
\end{aligned}
$$

Факторизация линейных операторов в задачах (55), которые соответствуют дискретной части основных уравнений $f_{j k}|\Psi\rangle=0$, является характеристическим свойством рассмотренных выше деформаций.

В координатном представлении, для которого $p_{1}=\partial_{x_{1}}, p_{2}=\partial_{x_{2}}, \operatorname{Tf}(x, n)=$ $f(x, n+1)$, линейные задачи (55) принимают вид

$$
\begin{gathered}
\left(\partial_{x_{1}}-(\ln \varphi)_{x_{1}}\right)\left(T \Psi-\left(\Psi_{x_{1}}-(\ln \widetilde{\Psi})_{x_{1}} \Psi\right)\right)=0 \\
\left(\partial_{x_{2}}-(\ln \varphi)_{x_{2}}\right)\left(T \Psi-\left(\Psi_{x_{1}}-(\ln \widetilde{\Psi})_{x_{1}} \Psi\right)\right)=0 \\
\left(T-\frac{T \varphi}{\varphi}\right)\left(T \Psi-\left(\Psi_{x_{1}}-(\ln \widetilde{\Psi})_{x_{1}} \Psi\right)\right)=0
\end{gathered}
$$

Из этих уравнений следует, что

$$
T \Psi=\Psi_{x_{1}}-(\ln \widetilde{\Psi})_{x_{1}} \Psi+\alpha \varphi,
$$

где $\alpha$ - произвольная константа. При $\alpha=0$ формула (57) есть не что иное, как стандартное преобразование волновой функции Буссинеска $\Psi$ под действием преобразования Дарбу (см., например, работу [14]). При $\alpha \neq 0$ функция $T \Psi-\alpha \varphi$ также является решением линейных задач (52) с потенциалами $T A, T D$.

Таким образом, теория преобразований Дарбу (по крайней мере, для уравнения Буссинеска) может быть полностью включена в теорию деформаций, представленную в настоящей работе. Однако ситуация с уравнением ВДВВ совсем иная. При редукции $C=1, G=N=0$ и затем $E=A, M=D$ получаются уравнения (46) и другие задачи, которые не слишком отличаются от задач для уравнения Буссинеска. Но в отличие от предыдущего случая одно из этих уравнений эквивалентно переопределенной системе нелинейных дифференциальных уравнений в частных производных, совместность которых весьма проблематична. Имеются основания предполагать, что для построения преобразования типа преобразования Дарбу для уравнения ВДВВ следует рассматривать алгебры более высоких размерностей $(N \geqslant 5)$.

7 Теоретическая и математическая физика, т. 159, № 3, 2009 г. 


\section{Список литературы}

[1] E. Witten, Nucl. Phys. B, 340:2-3 (1990), 281-332.

[2] R. Dijkgraaf, H. Verlinde, E. Verlinde, Nucl. Phys. B, 352:1 (1991), 59-86.

[3] B. Dubrovin, Nucl. Phys. B, 379:3 (1992), 627-689.

[4] B. Dubrovin, "Geometry of 2D topological field theories", Integrable Systems and Quantum Groups, Lecture Notes in Math., 1620, eds. M. Francaviglia, S. Greco, Springer, Berlin, 1996, 120-348.

[5] Y.I. Manin, Frobenius Manifolds, Quantum Cohomology and Moduli Spaces, Amer. Math. Soc. Colloq. Publ., 47, AMS, Providence, RI, 1999.

[6] C. Hertling, Frobenius Manifolds and Moduli Spaces for Singularities, Cambridge Tracts in Math., 151, Cambridge Univ. Press, Cambridge, 2002.

[7] B. G. Konopelchenko, F. Magri, Comm. Math. Phys., 274:3 (2007), 627-658.

[8] Б. Г. Конопельченко, Ф. Магри, ТМФ, 151:3 (2007), 439-457.

[9] B. G. Konopelchenko, F. Magri, Yano manifolds and coisotropic deformations, to appear.

[10] B. G. Konopelchenko, Quantum deformations of associative algebras and integrable systems, arXiv: 0802.3022.

[11] B. G. Konopelchenko, Discrete, q-difference deformations of associative algebras and integrable systems, arXiv: 0809.1938.

[12] В. Е. Захаров, ЖЭТФ, 62 (1972), 1745-1751.

[13] A. Givental, Mosc. Math. J., 3:2 (2003), 475-505.

[14] V. B. Matveev, M. A. Salle, Darboux Transformations and Solitons, Springer Ser. Nonlinear Dynam., Springer, Berlin, 1991. 